\title{
Ruptura de globo ocular y síndrome de Ehlers-Danlos VI
}

\section{Eye globe rupture and Ehlers-Danlos VI syndrome}

\author{
Rocío Villafuerte-de la Cruz ${ }^{1}$, Samantha Salinas-Longoria², Vianney Cortés-González ${ }^{1}$ y \\ Cristina Villanueva-Mendoza ${ }^{1 *}$
}

${ }^{1}$ Servicio de Genética; ${ }^{2}$ Servicio de Retina. Hospital Dr. Luis Sánchez Bulnes, Asociación para Evitar la Ceguera en México, IAP, Ciudad de México, México

\section{Resumen}

Introducción: El síndrome de Ehlers-Danlos (ED) comprende un grupo heterogéneo de enfermedades hereditarias del tejido conectivo; se reconocen 6 variedades principales. El tipo VI o variedad xifoescoliótica (ED-VI) es una variante rara, con modo de herencia autosómico recesivo, caracterizada clínicamente por hipotonía muscular neonatal, xifoescoliosis progresiva, hiperlaxitud articular, piel frágil e hiperelástica, osteopenia, ruptura de arterias, microcórnea y fragilidad de esclera, con riesgo de ruptura del globo ocular. El objetivo del reporte es la presentación de un paciente con síndrome de ED-Vt identificado por la ruptura del globo ocular posterior a un trauma leve. Caso clínico: Paciente masculino de 13 años que acude por disminución de la agudeza visual de $12 \mathrm{~h}$ de evolución posterior a trauma leve en ojo derecho. A la exploración se encontró: agudeza visual de percepción de luz que no discrimina color, hipotonía, hiposfagma y quemosis de $360^{\circ}$, córnea transparente de $9.8 \mathrm{~mm}$, cámara anterior estrecha, hipema 70\%, otras estructuras no eran valorables; el ultrasonido modo $B$ demostró pérdida de la continuidad escleral; en ojo izquierdo la agudeza visual era de 20/60, córnea de $9.8 \mathrm{~mm}$, esclera de tonalidad azul-grisácea, otras estructuras sin alteraciones. En la cirugía se describió esclera delgada y friable, así como músculos friables. En la valoración sistémica se encontró: piel hiperelástica, escoliosis toracolumbar, cicatrización atrófica e hiperlaxitud articular. Conclusiones: En todo paciente con ruptura del globo ocular asociado a trauma leve debe considerarse la posibilidad del síndrome de $E D$, por lo que será conveniente contar con historia clínica y valoración sistémica.

Palabras clave: Ehlers-Danlos. Xifoescoliosis. Ruptura de globo ocular. Microcórnea. Hiperlaxitud articular.

\section{Abstract}

Introduction: Ehlers-Danlos syndromes (EDS) comprises a clinically and genetically heterogeneous group of heritable disorders of connective tissue. Classification recognizes 6 main subtypes. The ED type VI or kyphoscoliotic type (ED-VI) is a rare autosomal recessive inherited disease characterized by severe neonatal muscular hypotonia, kyphoscoliosis, joint hypermobility, skin fragility, osteopenia, rupture of arteries, microcórnea and scleral fragility with increased risk of eye globe rupture. This is a case report of eye globe rupture in a patient with ED-VI. Case Report: A 13-year male patient consulted with decreased visual acuity after a mild trauma in right eye. Ophthalmic examination showed in right eye: Visual acuity of light perception with no color discrimination, hypotonic eye, hyposphagma and chemosis $360^{\circ}$, clear cornea with a horizontal diameter of $9.8 \mathrm{~mm}$, hyphema 70\%, others structures were not valuable; left eye with visual acuity of 20/60, corneal horizontal diameter of $9.8 \mathrm{~mm}$ and lightly blue-gray sclerae, no other abnormalities were found. Systemic evaluation demonstrated

\section{Correspondencia:}

*Cristina Villanueva-Mendoza

E-mail: villanuevacristina @ hotmail.com

Oftalmología. Publicado por Permanyer Mexico SA de CV. Este es un artículo Open Access bajo la licencia CC BY-NC-ND (http://creativecommons.org/licenses/by-nc-nd/4.0/).
Fecha de recepción: 23-05-2016

Fecha de aceptación: 30-06-2016

DOI: 10.1016/j.mexoft.2016.06.005
Disponible en internet: 02-07-2018 Rev Mex Oftalmol. 2018;92(4):209-213 www.rmo.com.mx

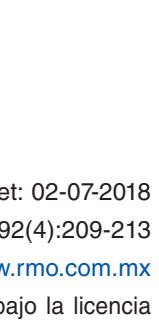


smooth and hyperelastic skin, scoliosis, atrophic scars and joint hypermobility. Conclusions: In every patient with eye globe rupture associated with mild trauma we must consider the diagnosis of EDS. It is necessary to have a complete medical record and systemic evaluation.

Key words: Ehlers-Danlos. Kyphoscoliosis. Eye globe rupture. Microcornea. Joint hypermobility.

\section{Introducción}

El síndrome de Ehlers-Danlos (ED) comprende un grupo heterogéneo de enfermedades hereditarias del tejido conectivo con manifestaciones variables en piel, ligamentos, articulaciones, vasos sanguíneos y algunos órganos internos ${ }^{1}$. En términos generales, los diferentes cuadros son el resultado de mutaciones en genes que codifican para las fibras colágenas o las enzimas involucradas en su síntesis ${ }^{1}$. Como consecuencia de esto se altera la fuerza y la estructura de la matriz extracelular en todos los tejidos y órganos del cuerpo. De acuerdo con la clasificación de Villefranche, se reconocen 6 variedades principales basadas en el fenotipo clínico, patrón hereditario y en el defecto bioquímico o molecular ${ }^{1,2}$. Además, se han descrito nuevas variantes $y$, en ocasiones, los fenotipos se sobreponen, lo que dificulta el diagnóstico ${ }^{1,3}$.

El síndrome de ED de tipo vi (ED-VI) o variedad xifoescoliótica es una variante rara (OMIM \# 225400), con modo de herencia autosómico recesivo y con una incidencia de 1:100,000 recién nacidos vivos ${ }^{1,3}$. Clínicamente se caracteriza por hipotonía muscular neonatal, retraso en el desarrollo motor, xifoescoliosis de inicio temprano, hiperlaxitud articular, osteopenia, piel frágil e hiperelástica, ruptura de arterias, microcórnea y fragilidad de esclera, con riesgo de ruptura del globo ocular. Está ocasionado por mutaciones en el gen PLOD1 (procolágena-lisil, 2 oxaglutarato 5-dioxigenasa). La enzima lisil-hidroxilasa 1 tiene la función de hidroxilar los residuos de lisina a hidroxilisina: estos residuos son precursores del proceso de entrecruzamiento de las fibras de colágena, mecanismo esencial para mantener la tensión en ellas ${ }^{4}$.

El objetivo del reporte es la presentación de un caso clínico con ruptura del globo ocular posterior a un trauma leve en un paciente con síndrome de ED-VI o variedad xifoescoliótica.

\section{Caso clínico}

Se trata de un paciente masculino de 13 años de edad, quien acude al Servicio de Urgencias de un hospital oftalmológico por disminución de la agudeza visual de $12 \mathrm{~h}$ de evolución posterior a trauma ocular. El paciente refirió haber tenido un trauma leve: «fue lastimado por el dedo de un compañero de escuela». A la exploración inicial se encontró en ojo derecho (OD) agudeza visual de percepción de luz que no discrimina color, hipoglobo, hipotonía, hiposfagma y quemosis de $360^{\circ}$, córnea transparente de $9.8 \mathrm{~mm}$, cámara anterior estrecha, hipema $70 \%$ y el resto no era valorable; en ojo izquierdo (OI) la agudeza visual era de 20/60, córnea de $9.8 \mathrm{~mm}$, esclera con tonalidad azul-grisácea, el resto estaba sin alteraciones. El estudio de ecografía modo $B$ en OD demostró: pliegues y engrosamiento coroideo generalizado y pérdida de la continuidad escleral en el sector temporal y superior (Fig. 1). El paciente fue intervenido y en la cirugía se describió esclera delgada y friable, músculos friables; el recto inferior no se localizó, se asumió que estaba desgarrado.

Posteriormente a la cirugía se solicitó valoración por genetista y se encontraron antecedentes importantes para la historia clínica. Es originario de una comunidad endogámica (menos de 2,000 habitantes) del Estado de México, los padres son sanos, no consanguíneos, tiene 2 hermanos sanos y una hermana de 21 años con diagnóstico de valvulopatía cardiaca. El embarazo fue de pretérmino, de 8 meses, se desconoce la causa. Hubo retraso en el desarrollo motriz, antecedentes de dislexia, pie plano, luxación de tobillo a los 8 años y ruptura de ligamentos de rodilla a los 9 . Cursa el primer año de secundaria con un rendimiento escolar bajo.

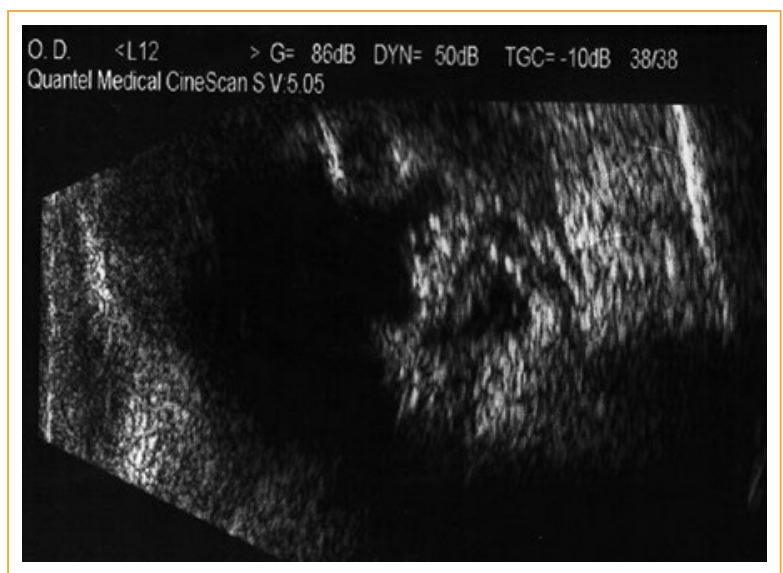

Figura 1. Ecografía modo B (OD): se observa pérdida de la continuidad escleral temporal y superior. 


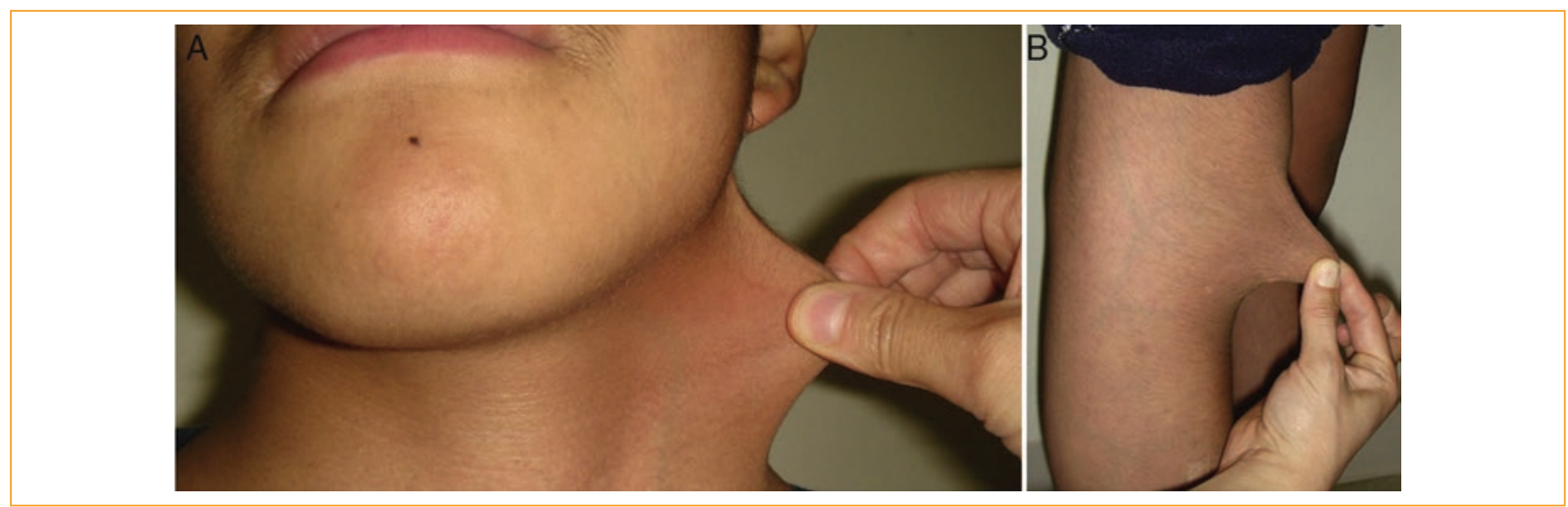

Figura 2. A: Piel hiperelástica de cuello B: y rodilla.

Hallazgos sistémicos: la piel era suave, hiperelástica (Fig. 2), presentaba pectus excavatum, escoliosis toracolumbar, diástasis de rectos anteriores; pliegue palmar transverso bilateral, ausencia de pliegue interfalángico medio y distal de $3 .^{\text {er }}$ y $4 .^{\circ}$ dedos bilateral; cicatrices atróficas «en papel de cigarro» en las piernas (Fig. 3), patelas hipermóviles, caída del arco plantar transverso, hallux valgus y del $2 .^{\circ}$ al $5 .^{\circ}$ ortejos «en garra", en forma bilateral. Se encontró hiperlaxitud articular generalizada con 7 puntos de la escala de Beighton (Fig. 4).

Las características oculares consistentes en fragilidad de los tejidos, escleras azules y microcórnea, así como los hallazgos sistémicos y el antecedente familiar en hermana con valvulopatía, probablemente asociada a enfermedad del tejido conectivo, fueron compatibles con el diagnóstico clínico de síndrome de ED-VI o variedad xifoescoliótica.

\section{Discusión}

El síndrome de ED-VI o variedad xifoescoliótica se caracteriza por hipotonía neonatal severa, xifoescoliosis progresiva, hiperlaxitud articular, fragilidad escleral y ruptura del globo ocular. Estas alteraciones se consideran criterios mayores para el diagnóstico. Otros hallazgos denominados criterios menores son: ruptura arterial, hábito marfanoide, osteopenia sin tendencia a fracturas patológicas, microcórnea, fragilidad de tejidos con cicatrización atrófica e historia familiar positiva para entidad autosómica recesiva ${ }^{1,5}$.

La hipotonía neonatal severa presente en casi todos los casos ocasiona a su vez retraso en el desarrollo motriz, posturas anormales y conlleva confusión en el diagnóstico, principalmente con miopatías o distrofias musculares de inicio temprano ${ }^{6}$. Por otro lado, la escoliosis se puede presentar desde los primeros meses de vida y es progresiva. Este dato, aunado a la presencia de la hipermovilidad articular y las luxaciones, puede ser importante para considerar el diagnóstico, ya que la ruptura del globo ocular es infrecuente. Vale la pena señalar que la hipermovilidad articular debe valorarse con base a la escala de Beighton (Tabla 1), en este caso se encontró un puntaje de 7 , que se consideró significativo para el diagnóstico ${ }^{1,7}$.

El diagnóstico inicial es clínico y se confirma mediante la determinación urinaria de la relación de piridinolinas: LP/HP (lisil-piridinolina/hidroxilisilpiridinolina), que se encuentra en un rango alto en los pacientes con ED-VI (rango normal es $0.20 \pm 0.03$, rango elevado en pacientes con ED-VI es $5.99 \pm 1.00$ ). También se debe buscar la deficiencia enzimática de lisil-hid̄oxilasa 1 en cultivo de fibroblastos y, finalmente, se debe confirmar mediante análisis molecular la mutación de PLOD1 1,4,8.

En el presente caso la sospecha diagnóstica se estableció con bases clínicas al identificar 4 criterios mayores: 1) hipotonía neonatal que ocasiona retraso en el desarrollo motor, 2) escoliosis, 3) hiperlaxitud articular y 4) fragilidad de esclera que ocasiona la ruptura del globo ocular ante un trauma leve, así como 3 criterios menores: 1) microcórnea, 2) fragilidad de tejidos con cicatrización atrófica y 3) historia familiar positiva para entidad autosómica recesiva. Como ya hemos señalado, lo que destaca clínicamente a ED-VI entre todos los subtipos de ED son las afecciones oftalmológicas severas, claramente presentes en este caso; sin embargo, la ruptura del globo ocular ocurre en una minoría de los pacientes ${ }^{9}$. 
Tabla 1. Escala de Beighton

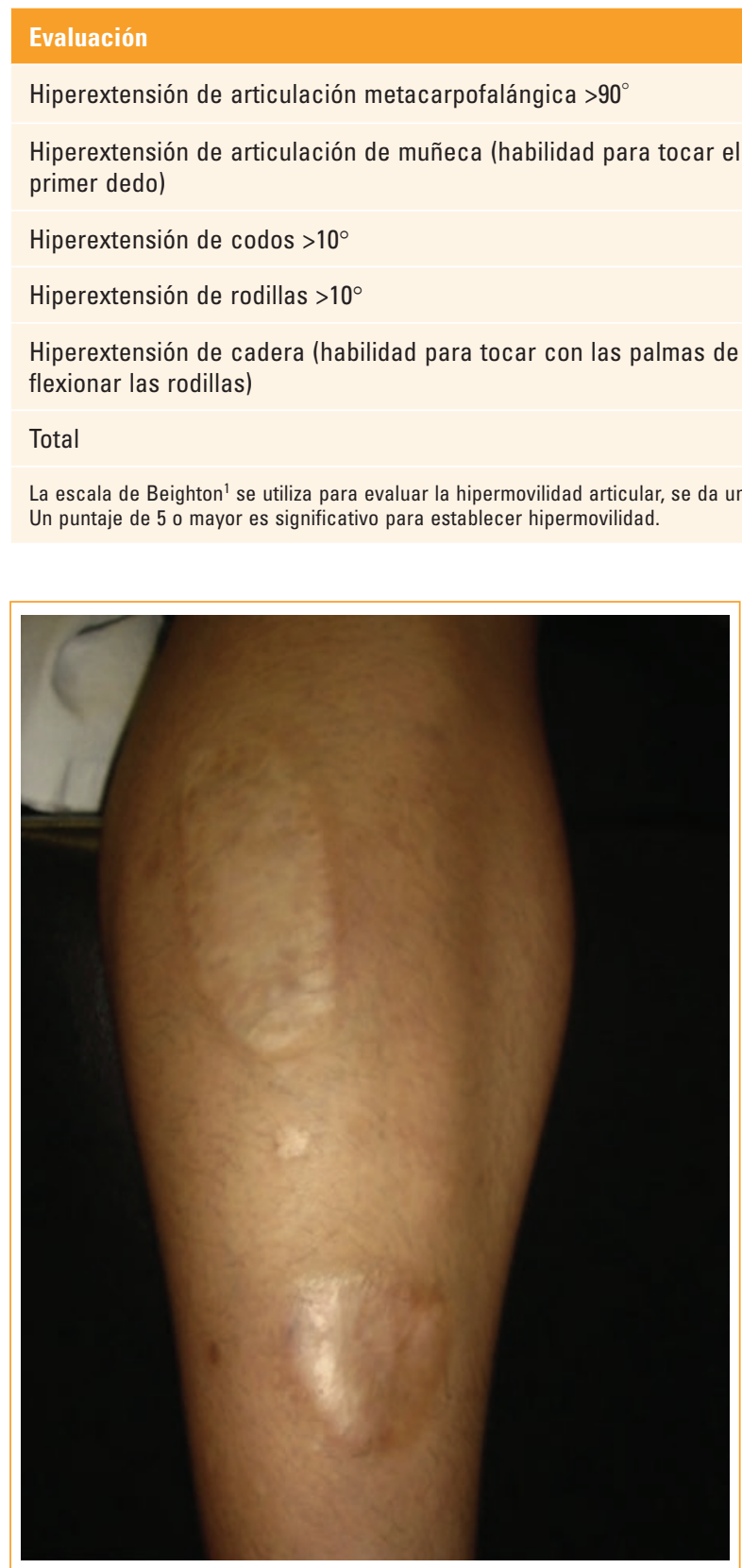

Figura 3. Cicatrices atróficas con aspecto de «papel de cigarro" en la piel de la pierna.

Ante la sospecha clínica o la confirmación del diagnóstico es importante realizar ecocardiograma con la finalidad de detectar alteraciones cardiovasculares, como puede ser la dilatación aórtica o el prolapso de la válvula mitral ${ }^{3}$ y realizar estudios como densitometría ósea por el riesgo de osteopenia ${ }^{6}$.

Dentro de los diagnósticos diferenciales que se deben considerar están:
1) Otras variedades de ED: por lo general, en todas las variedades hay hipermovilidad articular y cicatrización anormal, así mismo, hay riesgo de escoliosis. Sin embargo, en la variedad VI la escoliosis se inicia en los primeros meses de vida y es progresiva. En este caso la escoliosis no se manifestó en forma temprana y tampoco ha sido severa. Entonces, por las características oculares se considera el subtipo $\mathrm{VI}^{1-4}$. Existen 2 variedades de ED con patrón de herencia autosómico recesivo que comparten varias características con ED-VI: ED asociado a mutaciones en CHST14 (OMIM \#611776), también denominada variedad músculo-contractural, en la que se observan contracturas, aracnodactilia, pie equino varo, alteraciones craneofaciales, gastrointestinales y del tracto urinario; además, la relación urinaria de LP/ HP es normal. Esta variedad es difícil de diferenciar, dado que no se pudo realizar la determinación urinaria de LP/HP; sin embargo, por la ausencia de alteraciones craneofaciales en el paciente consideramos más probable la variedad xifoescoliótica o ED-VI1,3,4. Por último, ED asociado a mutaciones en FKBP14 (OMIM \#614557), en esta variedad también se encuentra normal la relación LP/HP e hipoacusia neurosensorial ${ }^{3}$.

2) Síndome de Marfan: aunque en esta entidad se puede encontrar hipermovilidad articular, en este caso no se consideró como diagnóstico diferencial, dada la ausencia de criterios, entre tos que se incluye la subluxación del cristalino, dato básico para la sospecha diagnóstica de síndrome de Marfan ${ }^{9,10}$.

3) Síndrome de córnea frágil, entidad autosómica recesiva, caracterizada por la ruptura de córnea secundaria a un trauma menor, queratocono o 


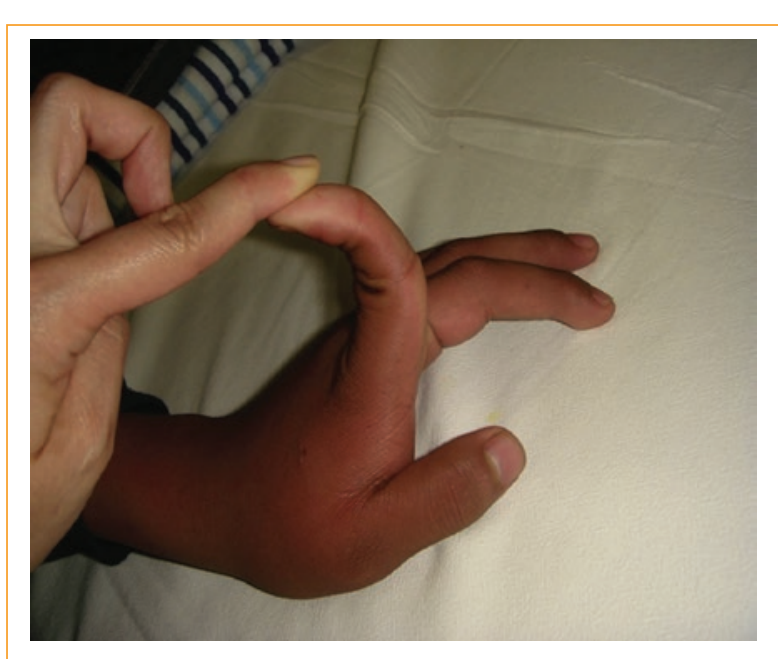

Figura 4. Hiperelasticidad de articulaciones interfalángicas en dedo índice.

queratoglobo, escleras azules, hiperelasticidad de piel e hiperlaxitud $\operatorname{articular}^{10}$. Las características oculares y sistémicas son semejantes en las 2 entidades, sin embargo, la ruptura del globo ocular únicamente está descrita en ED-VI. Además, la presencia de escoliosis y el antecedente de valvulopatía en una hermana correlacionan más con ED-VII1-13.

\section{Conclusiones}

En todo paciente con ruptura del globo ocular asociada a trauma leve debe considerarse la posibilidad del síndrome de ED, por lo que será conveniente contar con historia clínica y valoración sistémica.

Los hallazgos oftalmológicos y sistémicos en un paciente con sospecha de síndrome de ED pueden ayudar a establecer el subtipo específico, lo cual es importante para el seguimiento y el asesoramiento genético de cada caso.

El diagnóstico de esta enfermedad o de otra entidad subyacente es importante, ya que aumenta el riesgo de morbimortalidad de los pacientes.

\section{Responsabilidades éticas}

Protección de personas y animales. Los autores declaran que para esta investigación no se han realizado experimentos en seres humanos ni en animales.

Confidencialidad de los datos. Los autores declaran que han seguido los protocolos de su centro de trabajo sobre la publicación de datos de pacientes.

Derecho a la privacidad y consentimiento informado. Los autores han obtenido el consentimiento informado de los pacientes o sujetos referidos en el artículo. Este documento obra en poder del autor de correspondencia.

\section{Financiamiento}

Los autores no recibieron patrocinio para llevar a cabo este artículo.

\section{Conflicto de intereses}

Los autores declaran no tener ningún conflicto de intereses.

\section{Bibliografía}

1. Malfait F, de Paepe A. The Ehlers-Danlos syndrome. En: HalperJ, editor. Progress in heritable soft connective tissue diseases. Heidelberg, Nueva York, Londres: Springer Dordrecht; 2014. p. 129-43.

2. Beighton $P$, de Paepe A, Steinmann B, et al. Ehlers-Danlos syndromes: Revised nosology, Villefranche, 1997. Am J Med Genet.1998;77:31-7.

3. Sobey G. Ehlers-Danlos syndrome. How to diagnose and whento perform genetic tests. Arch Dis Child. 2015;100:57-61.

4. Giunta C, Randolph A, Steinmann B. Mutations analysis of the PLOD1 gene: An efficient multistep approach to the molecular diagnosis of the kyphoscoliotic type of Ehlers-Danlos syndrome (EDS VIA). Mol Genet Metab. 2005;86(1-2):269-76.

5. De Paepe A, Malfait F. Ehlers-Danlos syndrome, a disorder with many faces. Clin Genet. 2012;82:1-11.

6. Kariminejad A, Bozorgme h B, Khatami A, et al. Ehlers-Danlos syndrome type VI in a 17-year-old iranian boy with severe muscular weakness - a diagnostic challenge? Iran J Pediatr. 2010;20(3):358-62.

7. Yen J-L, Lin S-P, Chen M-R, et al. Clinical features of Ehlers-Danlos syndrome. J Formos Med Assoc. 2006;105(6):475-80.

8. Rohrbach M, Vandersteen A, Yis U, et al. Phenotypic variability of the kyphoscoliotic type of Ehlers-Danlos syndrome (EDS VI1): Clinical, molecular and biochemical delineation. OrphanetJ Rare Dis. 2011;6(46):1-9.

9. Callewaert B, Malfait F, Loeys B, et al. Ehlers-Danlos syndromes and Marfan syndrome. Best Pract Res Clin Rheumatol. 2007;22-1:165-89.

10. Zadeh N, Bernstein J, Niemi AK, et al. Ectopia lentis as the presenting and primary feature in Marfan syndrome. Am J Med Genet Part A. 2011;155:2661-8.

11. Royce P, Steinmann B, Vogel A, et al. Brittle cornea syndrome: An heritable connective tissue disorder distinct from Ehlers-Danlos syndrome type VI and fragilitas oculi, with spontaneous perforations of the eye, blue sclerae, red hair, and normal collagen lysyl hydroxylation. Eur $\mathrm{J}$ Pediatr. 1990;149:465-9.

12. Al-Hussain $\mathrm{H}$, Zeisberger $\mathrm{S}$, Huber $\mathrm{P}$, et al. Brittle cornea syndrome and its delineation from the kyphoscoliotic type of Ehlers Danlos syndrome (EDS VI): Report on 23 patients and review of the literature. Am J Med Genet. 2004;124A:28-34

13. Grahame R. Heritable disorders of connective tissue. Baillieres Best Pract Res Clin Rheumatol. 2000;14-2:345-61. 